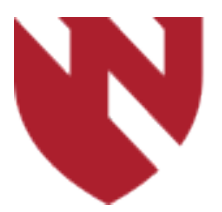

October 2021

\title{
Pediatric Orthopaedic Trauma: What Defines "Pediatric?"
}

\author{
Erin L. Stockwell \\ University of Nebraska Medical Center \\ Susan A. Scherl \\ University of Nebraska Medical Center \\ Maegen Wallace \\ University of Nebraska Medical Center \\ Matthew A. Halanski \\ University of Nebraska Medical Center \\ Brian P. Hasley \\ University of Nebraska Medical Center
}

Tell us how you used this information in this short survey.

Follow this and additional works at: https://digitalcommons.unmc.edu/gmerj

Part of the Higher Education Commons, and the Medicine and Health Sciences Commons

\section{Recommended Citation}

Stockwell, E. L., Scherl, S. A., Wallace, M., Halanski, M. A., , Hasley, B. P. Pediatric Orthopaedic Trauma: What Defines "Pediatric?". Graduate Medical Education Research Journal. 2021 Oct 04; 3(1). https://digitalcommons.unmc.edu/gmerj/vol3/iss1/19 


\section{Pediatric Orthopaedic Trauma: What Defines "Pediatric?"}

Creative Commons License

(c) 1 (1) (अ)

This work is licensed under a Creative Commons Attribution-Noncommercial-No Derivative Works 4.0 License. 


\section{Newsworthiness of Neurological Research}

Isha Snehal' ${ }^{1}$, Appaji Rayi ${ }^{2}$, Vineet Punia ${ }^{3}$

${ }^{1}$ University of Nebraska Medical Center, College of Medicine, Department of Neurological Sciences ${ }^{2}$ Ohio State Wexner Medical Center, Department of Neurology

${ }^{3}$ Epilepsy Center, Cleveland Clinic Foundation, Ohio

Mentor: Vineet Punia

Program: Neurology

Type: Original Research

Background: Informing the public of the latest scientific breakthroughs is an important result of research. Popular news media remains an impactful means of research dissemination. We, therefore, evaluated the factors that determine the newsworthiness of neurological research. Our objective was to evaluate news coverage received by neurological research and factors associated with its newsworthiness.

Methods: Original research articles from the year 2016 in the top 5 neurological journals, based on impact factor, were extracted. Individual research articles were reviewed for the sub-specialty, study design, funding source, continent and institute of origin. Dimensions, an online platform, was used to find the number of news and Twitter mentions and open access status. Articles receiving a news mention were considered 'newsworthy.' Logistic regression models for "newsworthiness" were performed.

Results: Of the 1050 research articles included, 551 (52.5\%) were newsworthy, with a median of 2 [inter-quartile range $(\mathrm{IQR})=1-10$ ] news mentions. Newsworthy articles were significantly more likely to have a higher number of tweets [Odds ratio $(\mathrm{OR})=1.04(1.03-1.05), \mathrm{p}<0.001]$ and open-access $(\mathrm{OR}=1.63(1.22-2.17), \mathrm{p}=$ $0.02)$. Newsworthiness was not associated with study design, article type, funding status, continent, or institute of origin. Publication in JAMA Neurology [OR $=2.86(1.39-$ $6.1), \mathrm{p}=0.005$ ] and the Lancet Neurology $[\mathrm{OR}=2.86(1.39-6.1), \mathrm{p}=0.005]$ affected newsworthiness. Articles on cognitive neurology were 22 times [OR $=22.62$ $(11.48-47.95), \mathrm{p}<0.001]$ more likely to be newsworthy.

Conclusion: Almost half of the research articles published in top neurology journals do not receive any news mention. Newsworthiness is associated with the popularity of research articles on social media. Studies on cognitive neurology seem to be of high interest in the news. Future research to understand the impact of newsworthiness on clinical outcomes is required.

https://doi.org/10.32873/unmc.dc.gmerj.3.1.038

\section{Pediatric Orthopaedic Trauma: What Defines "Pediatric?"}

Erin L. Stockwell', Susan A. Scherl ${ }^{1,2}$, Maegen J. Wallace ${ }^{1,2}$, Matthew A. Halanski ${ }^{1,2}$, Brian P. Hasley ${ }^{1,2}$ ${ }^{1}$ University of Nebraska Medical Center, College of Medicine, Department of Orthopaedic Surgery ${ }^{2}$ Children's Hospital and Medical Center, Department of Orthopaedic Surgery

Mentors: Brian Hasley, Susan Scherl

Program: University of Nebraska Medical Center Orthopaedic Surgery Residency Program

Type: Original Research

Background: Pediatric trauma patients have been shown to have better outcomes when treated at pediatric trauma centers, highlighting the importance of understanding the unique physiologic characteristics of pediatric patients. This suggests that pediatric orthopaedic trauma may be best defined physiologically, rather than by age. This study seeks to determine how pediatric orthopaedic trauma patients are defined and managed at leading pediatric orthopaedic departments.
Methods: A 19-question survey was sent to one designated member within the orthopaedic department of each of the Top 50 Children's Hospitals for Orthopaedics (per U.S. News and World Report 2019). Questions utilized multiple choice, yes/no, and open-ended response formats.

Results: Forty eight of the 50 surveys were completed. Pediatric orthopaedic trauma is defined by age at $78 \%$ of institutions. The mean upper-age limit was 18 years (range 14-26 years). All institutions treat simple and complex fractures in skeletally immature patients. Long bone fractures, complex periarticular fractures, and operative pelvic and acetabular fracture in skeletally mature patients were treated by $96 \%, 67 \%$ and $25 \%$ of respondents, respectively.
Conclusion: Nearly $80 \%$ of leading pediatric orthopaedic hospitals define orthopaedic trauma patients based on age. Most pediatric orthopaedists manage complex periarticular and long bone fractures in skeletally mature patients. Pediatric orthopaedist referral rates are increasing, and there is a growing concern for a shortage of pediatric orthopaedists. We challenge the norm of defining pediatric orthopaedic trauma patients based on an arbitrary age cutoff; defining based on physeal closure may improve outcomes while helping offload the burden of patient volume facing pediatric orthopaedists.

https://doi.org/10.32873/unmc.dc.gmerj.3.1.047

\section{The Isolated Fibula Fracture: Successful Outcomes with Non-operative, Immediate Weight Bearing Despite Stress Positive Radiographs Phillip Thomas ${ }^{1}$, Leonid Grossman², Sara Putnam¹, Justin Siebler ${ }^{1}$, Elizabeth Lyden ${ }^{3}$, Matthew Mormino \\ ${ }^{1}$ University of Nebraska Medical Center, College of Medicine, Department of Orthopedic Surgery \\ ${ }^{2}$ University of Nebraska Medical Center, College of Public Health, Department of Biostatistics \\ ${ }^{3}$ Panorama Orthopedics and Spine Center}

Mentor: Matthew Mormino

Program: Orthopedic Surgery

Type: Original Research
Background: Treatment of isolated distal fibula fractures relies on identifying whether a fracture is stable or unstable. Stress radiographs are the current gold standard for evaluating stability in ankle fractures. We present a novel method of evaluation for ankle fracture stability and treatment using an immediate weight-bearing (WB) protocol. 